\title{
Efeito da Má Especificação de Modelos nas Combinações de Previsão em Séries Temporais com Longa Dependência
}

\author{
Cleber Bisognin ${ }^{1}$ \\ Letícia Menegotto ${ }^{2}$ \\ Liane Werner ${ }^{3}$
}

Resumo: Ao modelarmos processos estocásticos, é possível cometermos equívocos no tipo de processo ou mesmo no número de parâmetros do processo a ser ajustado em determinada série. O objetivo deste trabalho é verificar a influência da má especificação de modelos nas previsões e nas combinações de previsões através das medidas de acurácia quando a série apresenta a propriedade de longa dependência, uma vez que comumente séries temporais que apresentam esta propriedade são confundidas com séries temporais não estacionárias. Utilizando a técnica de Monte Carlo serão realizadas simulações para verificar esta influência, onde será calculada a média das medidas de acurácia calculadas para cada modelo a ser verificado. Analisando as simulações de Monte Carlo, observamos que na grande maioria das vezes as combinações de previsões têm melhor capacidade preditiva que o próprio modelo a partir do qual a série for gerada - neste caso, $\operatorname{ARFIMA}(p, d, q)$. Finalmente será feita uma aplicação a dados reais, na qual será analisada a série temporal do valor do ativo do Banco Bradesco SA na hora do fechamento da bolsa de valores.

Palavras-chave: Combinação de Previsões, Modelagem Estatística, Previsões, Longa Dependência, Má Especificação de Modelos.

\section{Introdução}

De acordo com Abraham e Ledolter (2009), o ser humano está sempre fazendo previsões, que consiste em uma atividade indispensável no planejamento, na definição da estratégia e na tomada de decisões orientadas para o futuro, tanto em nível individual como em nível organizacional.

Uma vez que previsões envolvem eventos futuros e estes, por sua vez, envolvem a incerteza, tem-se que as previsões, em geral, não são perfeitas. O objetivo, ao realizarmos uma previsão, é reduzir o erro da mesma (Abraham e Ledolter, 2009). Para produzir uma previsão que apresente um erro pequeno, é necessário utilizar uma técnica de previsão adequada, seja por meio de um

\footnotetext{
${ }^{1}$ UFRGS - Universidade Federal do Rio Grande do Sul. Email: cbisognin@ufrgs.br

${ }^{2}$ UFRGS - Universidade Federal do Rio Grande do Sul. Email: leticia.menegotto@gmail.com

${ }^{3}$ UFRGS - Universidade Federal do Rio Grande do Sul. Email: liane.werner@ufrgs.br
} 
modelo ou uma combinação de previsões oriundas de várias técnicas de previsão, e para tanto, é preciso obter critérios de acurácia (Werner, 2005).

Conforme Morettin e Toloi (2006), uma das suposições mais frequentes que se faz a respeito de uma série temporal é que se desenvolva no tempo, aleatoriamente ao redor de uma média constante, refletindo alguma forma de equilíbrio estável (estacionaridade). Todavia, a maior parte das séries que se encontra na prática apresenta alguma forma de não estacionariedade, pois mudam suas características estocásticas ao longo do tempo de observação, sendo conhecidas por séries não estacionárias. Segundo Box e Jenkins (1976) é possível obter séries estacionárias pela diferenciação $(d)$, valor este assumido como número inteiro. Uma diferenciação fracionária é o caso geral do processo de diferenciação, modelos que usam este procedimento são conhecidos por modelos de longa dependência. Nas últimas décadas, tem ocorrido grande interesse no estudo de séries temporais com longa dependência, que iniciaram com os estudos de Hurst em 1951 quanto investigava a série temporal dos níveis mensais do rio Nilo (Bisognin, 2007). Segundo Lima et al. (2007) modelos de longa dependência são capazes de produzir previsões com menor erro quadrado médio, uma importante medida de acurácia.

Quando as medidas de acurácia são boas, acreditamos que um modelo adequado foi encontrado. Porém, é preciso ter cuidado na especificação do modelo. Para Queiroz (2016) a solução de problemas estatísticos está baseada na teoria da máxima verossimilhança, que tem como suposição básica de que o modelo escolhido para analisar os dados é, de fato, o modelo gerador destes. Quando isso não acontece, ou seja, quando ocorre uma má especificação do modelo, utilizar os procedimentos inferenciais usuais pode resultar em conclusões errôneas, gerando interpretações equivocadas.

Frente a isto, o objetivo deste trabalho é verificar a influência da má especificação de modelos na previsão e nas combinações de previsões através das medidas de acurácia, tendo como modelo gerador uma série que apresenta longa dependência. Tal objetivo deve-se ao fato que algumas séries temporais, podem ser tratadas como estacionária ou não estacionárias, ou seja, quando analisamos tais series com testes de raiz unitária, o p-valor de um teste é aproximadamente 0.1 e de outro menos que 0.05, como é o caso da série temporal do valor do ativo do Banco Bradesco SA na hora do fechamento da bolsa de valores. Maiores detalhes serão abordados na Seção 4.

\section{Técnicas de Previsão}

Nesta seção apresentamos os modelos utilizados para análise e previsão de séries temporais. Serão utilizados os modelos $\operatorname{ARMA}(p, q), \operatorname{ARIMA}(p, d, q), \operatorname{ARFIMA}(p, d, q)$ e suavização exponencial, além de três métodos para realizar combinações de previsões, a saber: variância mínima, 
por regressão e média aritmética.

Inicialmente definimos os processos $\operatorname{ARIMA}(p, d, q)$ proposto por Box e Jenkins (1976).

Definição 1. Seja $\left\{X_{t}\right\}_{t \in \mathbb{Z}}$ um processo estocástico satisfazendo a equação

$$
\phi(\mathcal{B})(1-\mathcal{B})^{d}\left(X_{t}-\mu\right)=\theta(\mathcal{B}) \varepsilon_{t},
$$

onde $\mu$ é a média do processo, $\left\{\varepsilon_{t}\right\}_{t \in \mathbb{Z}}$ é o processo ruído branco, $\mathcal{B}$ é o operador defasagem ou de retardo, isto é, $\mathcal{B}^{j}\left(X_{t}\right)=X_{t-j}$, para $j \in \mathbb{N}, \phi(\cdot)$ e $\theta(\cdot)$ são os polinômios de ordem $p$ e $q$, respectivamente, definidos por

$$
\phi(z)=\sum_{\ell=0}^{p}\left(-\phi_{\ell}\right) z^{\ell} \quad \text { e } \theta(z)=\sum_{m=0}^{q}\left(-\theta_{m}\right) z^{m}
$$

onde $\phi_{\ell}, 1 \leqslant \ell \leqslant p$ e $\theta_{m}, 1 \leqslant m \leqslant q$, são constantes reais e $\phi_{0}=-1=\theta_{0}$. Então, $\left\{X_{t}\right\}_{t \in \mathbb{Z}}$ é um processo auto-regressivo integrado de média móvel de ordem $(p, d, q)$, denotado por $\operatorname{ARIMA}(p, d, q)$, onde $d \in \mathbb{Z} \geqslant$ é o grau de diferenciação.

Observação 1. Na Definição 1, quando $d=0$, temos os processos $\operatorname{ARMA}(p, q)$.

Durante as últimas décadas, houve muito interesse em estudar séries temporais com a propriedade de longa dependência. Utilizando a definição de longa dependência, Granger e Joyeux (1980), Hosking (1981), Hosking (1984) e Geweke e Porter-Hudak (1983) apresentam os processos auto-regressivos fracionariamente integrados de média móvel $(\operatorname{ARFIMA}(p, d, q))$ como um exemplo de processos com a característica de longa dependência. A seguir definimos os processos $\operatorname{ARFIMA}(p, d, q)$.

Definição 2. Seja $\left\{X_{t}\right\}_{t \in \mathbb{Z}}$ um processo estocástico satisfazendo a equação

$$
\phi(\mathcal{B})(1-\mathcal{B})^{d}\left(X_{t}-\mu\right)=\theta(\mathcal{B}) \varepsilon_{t},
$$

onde $\mu$ é a média do processo, $\left\{\varepsilon_{t}\right\}_{t \in \mathbb{Z}}$ é o processo ruído branco, $\mathcal{B}$ é o operador de defasagem ou de retardo, isto é, $\mathcal{B}^{j}\left(X_{t}\right)=X_{t-j}$, para todo $j \in \mathbb{N}, \phi(\cdot)$ e $\theta(\cdot)$ são os polinômios de ordem $p$ e $q$, respectivamente, definidos por

$$
\phi(z)=\sum_{\ell=0}^{p}\left(-\phi_{\ell}\right) z^{\ell}, \quad \theta(z)=\sum_{m=0}^{q}\left(-\theta_{m}\right) z^{m},
$$

onde $\phi_{\ell}, 1 \leqslant \ell \leqslant p$ e $\theta_{m}, 1 \leqslant m \leqslant q$, são constantes reais e $\phi_{0}=-1=\theta_{0}$. Então, $\left\{X_{t}\right\}_{t \in \mathbb{Z}}$ é um processo auto-regressivo fracionariamente integrado de média móvel de ordem $(p, d, q)$ com média $\mu$, denotado por $\operatorname{ARFIMA}(p, d, q)$, onde $d$ é o grau de diferenciação fracionário.

Hosking (1981) demonstra que os processos $\operatorname{ARFIMA}(p, d, q)$ são estacionários se $d<\frac{1}{2}$ e as 
raízes da equação $\phi(z)=0$ estão fora do círculo unitário; e é inversível se $d>-\frac{1}{2}$ e as raízes da equação $\theta(z)=0$ estão fora do círculo unitário.

Além destes, os modelos de suavização exponencial, devido a sua simplicidade, facilidade de ajustes e boa acurácia, são os mais utilizados frente a outras técnicas de previsão, segundo Pellegrini (2000). Como assumem que os valores extremos da série são flutuações aleatórias, o propósito destes modelos é identificar um padrão básico na série temporal a ser analisada (Morettin e Toloi, 2006). Estes modelos valorizam mais as últimas observações na série temporal através da ponderação exponencial das mesmas, de acordo com a proximidade ao período da previsão $h$. Os métodos mais tradicionais de suavização exponencial são: (i) a suavização exponencial simples, para séries que apresentam apenas variações em torno de um nível; (ii) o modelo linear de Holt, para as séries que apresentam a componente de tendência e (iii) os modelos de Holt-Winters, quando a série apresenta tanto o componente de tendência quanto o componente sazonal (Makridakis et al., 1998).

A seguir definimos os modelos lineares de Holt. Maiores detalhes sobre estes modelos e os de Holt-Winters podem ser encontrados em Makridakis et al. (1998) e em Morettin e Toloi (2006).

\section{Modelos Lineares de Holt}

Seja uma série temporal $\left\{X_{t}\right\}_{t=1}^{n}$. No caso dos modelos lineares de Holt consideramos que tal série é formada pela soma do nível, tendência e um erro aleatório, como segue:

$$
X_{t}=L_{t}+T_{t}+\varepsilon_{t}, \quad \text { para } t=1, \cdots, n .
$$

As estimativas do nível da série no tempo $t$, denotado por $L_{t}$ e da tendência, denotada por $T_{t}$, são dadas, respectivamente por

$$
\begin{aligned}
L_{t} & =\alpha X_{t}+(1-\alpha)\left(L_{t-1}+T_{t-1}\right) \\
T_{t} & =\beta\left(L_{t}-L_{t-1}\right)+(1-\beta) T_{t-1},
\end{aligned}
$$

onde $\alpha$ é o coeficiente de ponderação exponencial do nível $(0 \leqslant \alpha \leqslant 1)$ e $\beta$ é o coeficiente de ponderação exponencial da tendência $(0 \leqslant \beta \leqslant 1)$.

As previsões $h$ passos a frente são dadas por

$$
\widehat{X}_{t}(h)=L_{t}+h T_{t}
$$

A notação $\widehat{X}_{t}(h)$ indica a previsão de origem $t$ e horizonte $h \geqslant 1$.

Além destes modelos, um método comumente utilizado para melhorar a acurácia das previsões é a combinação de previsões. Segundo Costantini e Pappalardo (2010), este método consiste em 
utilizar um mecanismo para captar os diversos fatores que afetam cada técnica de previsão individual usada como base na obtenção da previsão combinada.

O método da variância mínima, proposto por Bates e Granger (1969) consiste em realizar a combinação linear de duas previsões com diferentes pesos. Neste método a combinação das previsões é obtida atribuindo-se um peso para cada uma das previsões individuais que serão combinadas. Sua estrutura é apresentada conforme equação (9).

$$
F_{c}=w F_{1}+(1-w) F_{2}
$$

onde $w$ é o peso atribuído a previsão de menor variância e $F_{1}$ e $F_{2}$ são as previsões individuais a serem combinadas.

Para a obtenção dos pesos descritos na equação (9) é interessante atribuir menor peso às previsões de maior variabilidade nos erros e considerar a correlação existente entre os erros das duas previsões individuais realizadas. O peso para a previsão com menor variabilidade nos erros é obtido conforme equação (10).

$$
w=\frac{\sigma_{2}^{2}-\rho \sigma_{1} \sigma_{2}}{\sigma_{1}^{2}+\sigma_{2}^{2}-2 \rho \sigma_{1} \sigma_{2}} .
$$

onde $\rho$ é o valor da correlação linear entre os erros das previsões obtidas em $F_{1}$ e $F_{2}, \sigma_{1}^{2}$ é a variância dos erros de previsão de $F_{1}$ e $\sigma_{2}^{2}$ é a variância dos erros de previsão de $F_{2}$.

O método mais popular de combinação de previsões individuais é a média aritmética, pois além de ser um dos métodos mais conhecidos é fácil de calcular. Segundo De Menezes et al. (2000), uma possível resposta para o sucesso da média pode estar associada à instabilidade dos pesos ao longo do tempo na matriz de covariância dos erros das previsões individuais.

Um fato que chamou a atenção de Granger e Ramanathan (1984) é que a combinação de previsões poderia ser uma forma estruturada de regressão, utilizando o Método dos Mínimos Quadrados Ordinários (MQO), tendo a previsão combinada como variável resposta e as previsões individuais como variáveis explicativas.

De acordo com Makridakis et al. (1998), a palavra acurácia refere-se a habilidade do modelo ou da combinação em reproduzir os dados que já são conhecidos (qualidade do ajuste). Porém para optar qual técnica de previsão - individual ou combinação - é a mais adequada, faz-se necessário obter medidas de acurácia. Neste trabalho iremos utilizar as medidas de acurácia Root Mean Squared Error (RMSE), erro médio absoluto (MAE), erro percentual médio (MPE), erro percentual médio absoluto (MAPE) e erro médio de previsão (ME). 


\section{Simulações de Monte Carlo}

Nesta seção serão apresentados os resultados tendo como base os procedimentos metodológicos de simulação de Monte Carlo. O procedimento consiste em gerar séries temporais (amostras) dos processos $\operatorname{ARFIMA}(p, d, q)$, com $0<d<0.5$ (ver Definição 2). As séries temporais foram geradas utilizando a rotina fracdiff.sim, do pacote fracdiff do software R 3.4.0. Após foram ajustados as séries temporais geradas processos $\operatorname{ARFIMA}(p, d, q)$, utilizando a rotina arfima, do pacote forecast, processos $\operatorname{ARIMA}(p, d, q)$ e $\operatorname{ARMA}(p, q)$, utilizando a rotina auto.arima, também do pacote forecast, e o modelo de suavização exponencial, mais conhecido como Modelo Linear de Holt, utilizando a rotina HoltWinters, do pacote stats.

No caso dos processos $\operatorname{ARFIMA}(p, d, q)$, a rotina seleciona automaticamente os valores de $p$ e $q$ usando o algoritmo Khandakar e Hyndman (2008) e o algoritmo de Haslett e Raftery (1989), que é baseado no método da máxima verossimilhança, para estimar o parâmetros incluindo o parâmetro de longa dependência $d$.

Para a estimação dos parâmetros dos processos dos modelos ARIMA e ARMA foi utilizado a rotina auto.arima que calcula a verossimilhança exata via representação de Estado de Espaço do modelo enquanto as inovações são encontradas via Filtro de Kalmann. A estimação dos coeficientes dos polinômios é baseada em Gardner et al. (1980).

Para os modelos de suavização exponencial foi utilizado a rotina HoltWinters. A função tenta encontrar valores ótimos para $\alpha$, e/ou $\beta$ minimizando o erro quadrado de previsão de um passo à frente quando nenhum dos parâmetros de suavização é informado pelo usuário.

Após ajuste de modelos e teste de resíduos (rotina Box.test) foram calculadas as previsões dos $n$ valores da série temporal gerada e também serão aplicadas as técnicas de combinação previsão de variância mínima, média aritmética e por regressão, como base nos modelos individuais previamente obtidos combinados dois a dois. As técnicas de combinação de previsão foram implementadas no mesmo software.

Calculadas as previsões, o próximo passo é calcular as medidas de acurácia ME (média dos erros de previsão), RMSE (raiz do erro médio quadrático), MAE (erro médio absoluto de previsão), MPE (percentual médio de erro) e pelo MAPE (percentual médio absoluto de erro). As medidas foram calculadas utilizando-se a rotina accuracy do pacote forecast.

As Tabelas 1 a 5 contemplam os resultados de simulação de Monte Carlo para o procedimento descrito acima, e apresentam as médias das medidas de acurácia, para as $r e=1000$ replicações, das previsões utilizando os modelos e os três tipos de combinação de previsão. Foram geradas séries temporais, para cinco composições dos seguintes valores dos parâmetros $d=0.3, p \in\{0,1\}$, 
$\phi_{1} \in\{-0.8,0.8\}, q \in\{0,1\}, \theta_{1} \in\{-0.2,0.2\}, \operatorname{com} n=1000$.

Analisando a Tabela 1, quando geramos amostras dos processos $\operatorname{ARFIMA}(p, d, q), \operatorname{com} d=$ $0.3, p=1, \phi_{1}=-0.8$ e $q=0$, concluímos que os modelos com menor ME são os modelos $\operatorname{ARIMA}(p, d, q)$, a combinação de previsões de variância mínima utilizando os modelos $\operatorname{ARMA}(p, q)$ e Holt, e a combinação de previsões por regressão e por média dos modelos $\operatorname{ARIMA}(p, d, q)$ e $\operatorname{ARMA}(p, q)$. Com menor RMSE, MAE e MAPE é a combinação de previsões de variância mínima utilizando os modelos $\operatorname{ARIMA}(p, d, q)$ e Holt. Já o menor MPE foi encontrado na combinação de previsões por variância mínima dos modelos $\operatorname{ARIMA}(p, d, q)$ e Holt.

Pela análise da Tabela 2, quando geramos amostras dos processos $\operatorname{ARFIMA}(p, d, q)$, quando $d=0.3, p=1, \phi_{1}=0.8$ e $q=0$, concluímos que o modelo com menor ME é o modelo $\operatorname{ARMA}(p, q)$ e a combinação de previsões por média dos modelos $\operatorname{ARFIMA}(p, d, q)$ e $\operatorname{ARMA}(p, q)$. O modelo $\operatorname{ARMA}(p, q)$ possui menor RMSE e MAPE, enquanto o modelo $\operatorname{ARFIMA}(p, d, q)$ possui menor MAE e a combinação de previsões por variância mínima utilizando os modelos $\operatorname{ARIMA}(p, d, q)$ e Holt possui menor MPE.

Pela Tabela 3, quando geramos amostras dos processos $\operatorname{ARFIMA}(p, d, q)$, quando $d=0.3$, $p=0, q=1$ e $\theta_{1}=0.2$, verificamos que o modelo $\operatorname{ARIMA}(p, d, q)$ e a combinação de previsões por regressão dos modelos $\operatorname{ARFIMA}(p, d, q)$ e $\operatorname{ARMA}(p, q)$ possuem menor ME, a combinação de previsões por média dos modelos $\operatorname{ARFIMA}(p, d, q)$ e Holt possui menores RMSE, MAE e MAPE, enquanto a combinação de previsões por regressão dos modelos $\operatorname{ARIMA}(p, d, q)$ e Holt possui menor MPE.

Através da Tabela 4, quando geramos amostras dos processos $\operatorname{ARFIMA}(p, d, q), \operatorname{com} d=0.3$, $p=0, q=1$ e $\theta_{1}=-0.2$, constatamos que o modelo Holt, a combinação de previsões por variância mínima dos modelos $\operatorname{ARFIMA}(p, d, q)$ e Holt e a combinação de previsões por regressão dos modelos $\operatorname{ARFIMA}(p, d, q)$ e $\operatorname{ARMA}(p, q)$ e dos modelos $\operatorname{ARMA}(p, q)$ e Holt possuem menor ME. A combinação de previsões por média dos modelos $\operatorname{ARFIMA}(p, d, q)$ e Holt possui menor RMSE, MAE, MPE e MAPE.

Analisando a Tabela 5 , quando geramos amostras dos processos $\operatorname{ARFIMA}(p, d, q)$, quando $d=$ $0.3, p=1, \phi_{1}=0.8, q=1$ e $\theta_{1}=-0.2$, observamos que o modelo $\operatorname{ARIMA}(p, d, q)$, a combinação de previsões por variância mínima dos modelos $\operatorname{ARIMA}(p, d, q)$ e Holt e a combinação de previsões por média dos modelos $\operatorname{ARIMA}(p, d, q)$ e $\operatorname{ARMA}(p, q)$ e dos modelos $\operatorname{ARMA}(p, q)$ e Holt possuem menor ME. A combinação de previsões por média dos modelos $\operatorname{ARFIMA}(p, d, q)$ e $\operatorname{ARMA}(p, q)$ possui menor RMSE, a combinação de previsões por regressão dos modelos $\operatorname{ARFIMA}(p, d, q)$ e $\operatorname{ARMA}(p, q)$ possui menor MAE, enquanto os menores valores de MPE e MAPE ocorrem na 
combinação de previsões por variância mínima dos modelos $\operatorname{ARIMA}(p, d, q)$ e $\operatorname{ARMA}(p, q)$.

Tabela 1: Medidas de Acurácia para séries temporais geradas a partir dos processos $\operatorname{ARFIMA}(p, d, q)$, quando $d=0.3, p=1, \phi_{1}=-0.8, q=0$ e $n=1000$.

\begin{tabular}{lccccc}
\hline Modelos Ajustados & ME & RMSE & MAE & MPE & MAPE \\
\hline ARFIMA & -0.0002 & 0.9978 & 0.7963 & -1.0660 & 8.2262 \\
ARIMA & $\mathbf{0 . 0 0 0 0}$ & 1.0024 & 0.7996 & -1.0519 & 8.2601 \\
ARMA & -0.0001 & 0.9974 & 0.7959 & -1.0669 & 8.2300 \\
Holt & -0.0010 & 1.6295 & 1.2831 & -2.2506 & 13.3495 \\
\hline \multicolumn{5}{c}{ Combinação de Previsões - Variância } & Mínima \\
\hline ARFIMA/ARIMA & 0.0006 & 0.9977 & 0.7962 & -1.0573 & 8.2265 \\
ARFIMA/ARMA & 0.0019 & 0.9989 & 0.7974 & -1.0457 & 8.2360 \\
ARFIMA/Holt & -0.0002 & 0.7434 & 0.5933 & -0.8310 & 6.1339 \\
ARIMA/ARMA & 0.0003 & 1.0000 & 0.7977 & -1.0521 & 8.2406 \\
ARIMA/Holt & -0.0006 & $\mathbf{0 . 7 3 2 8}$ & $\mathbf{0 . 5 8 4 7}$ & $\mathbf{- 0 . 8 1 3 7}$ & $\mathbf{6 . 0 4 5 5}$ \\
ARMA/Holt & $\mathbf{0 . 0 0 0 0}$ & 0.7434 & 0.5934 & -0.8270 & 6.1391 \\
\hline \multicolumn{5}{c}{ Combinação de Previsões - Regressão } \\
\hline ARFIMA/ARIMA & 0.0002 & 0.9958 & 0.7943 & -1.0556 & 8.2047 \\
ARFIMA/ARMA & -0.0001 & 0.9956 & 0.7942 & -1.0637 & 8.2093 \\
ARFIMA/Holt & -0.0002 & 0.7418 & 0.5919 & -0.8328 & 6.1254 \\
ARIMA/ARMA & $\mathbf{0 . 0 0 0 0}$ & 0.9954 & 0.7942 & -1.0586 & 8.2046 \\
ARIMA/Holt & 0.0015 & 0.7342 & 0.5857 & -0.7952 & 6.0571 \\
ARMA/Holt & 0.0001 & 0.7443 & 0.5941 & -0.8277 & 6.1465 \\
\hline \multicolumn{7}{c}{ Combinação de Previsões - Média } \\
\hline ARFIMA/ARIMA & -0.0001 & 0.9974 & 0.7962 & -1.0598 & 8.2289 \\
ARFIMA/ARMA & 0.0001 & 0.9959 & 0.7948 & -1.0633 & 8.2179 \\
ARFIMA/Holt & 0.0002 & 1.0301 & 0.8157 & -1.4743 & 8.5070 \\
ARIMA/ARMA & $\mathbf{0 . 0 0 0 0}$ & 0.9963 & 0.7953 & -1.0566 & 8.2141 \\
ARIMA/Holt & -0.0004 & 1.0273 & 0.8145 & -1.4675 & 8.4935 \\
ARMA/Holt & -0.0007 & 1.0314 & 0.8175 & -1.4824 & 8.5233 \\
\hline
\end{tabular}

Fonte: Autores.

Tabela 2: Medidas de Acurácia para séries temporais geradas a partir dos processos $\operatorname{ARFIMA}(p, d, q)$, quando $d=0.3, p=1, \phi_{1}=0.8, q=0$ e $n=1000$.

\begin{tabular}{|c|c|c|c|c|c|}
\hline Modelos Ajustados & $\mathrm{ME}$ & RMSE & MAE & MPE & MAPE \\
\hline ARFIMA & -0.0001 & 0.9976 & 0.7960 & -1.1196 & 12.0266 \\
\hline ARIMA & 0.0004 & 1.0125 & 0.8079 & -1.4654 & 13.3490 \\
\hline ARMA & 0.0000 & 0.9973 & 0.7961 & -1.3920 & 11.8463 \\
\hline Holt & -0.0010 & 1.0461 & 0.8348 & -1.1510 & 12.1322 \\
\hline \multicolumn{6}{|c|}{ Combinação de Previsões - Variância Mínima } \\
\hline ARFIMA/ARIMA & -0.0001 & 0.9984 & 0.7964 & -3.5889 & 15.1945 \\
\hline ARFIMA/ARMA & 0.0024 & 1.0021 & 0.7995 & -1.7194 & 12.4937 \\
\hline ARFIMA/Holt & -0.0002 & 1.1847 & 0.9454 & -1.4550 & 14.4596 \\
\hline ARIMA/ARMA & 0.0002 & 1.0137 & 0.8085 & -1.8338 & 13.2070 \\
\hline ARIMA/Holt & -0.0004 & 1.1928 & 0.9514 & 0.7557 & 15.8453 \\
\hline ARMA/Holt & -0.0001 & 1.1854 & 0.9461 & -8.9787 & 22.2009 \\
\hline \multicolumn{6}{|c|}{ Combinação de Previsões - Regressão } \\
\hline ARFIMA/ARIMA & 0.0032 & 0.9992 & 0.7973 & -4.9606 & 15.2409 \\
\hline ARFIMA/ARMA & 0.0002 & 0.9984 & 0.7968 & -3.2177 & 14.9527 \\
\hline ARFIMA/Holt & 0.0007 & 1.1836 & 0.9445 & 3.6867 & 19.9684 \\
\hline ARIMA/ARMA & 0.0021 & 0.9987 & 0.7971 & 1.2228 & 14.7097 \\
\hline ARIMA/Holt & 0.0435 & 1.1887 & 0.9487 & -1.6423 & 14.7383 \\
\hline ARMA/Holt & -0.0007 & 1.1862 & 0.9467 & -2.3120 & 14.3208 \\
\hline \multicolumn{6}{|c|}{ Combinação de Previsões - Média } \\
\hline ARFIMA/ARIMA & 0.0003 & 1.0018 & 0.7994 & -2.1029 & 12.3018 \\
\hline ARFIMA/ARMA & 0.0000 & 0.9990 & 0.7972 & -2.7820 & 13.8283 \\
\hline ARFIMA/Holt & -0.0001 & 1.1069 & 0.8836 & -1.8229 & 13.9846 \\
\hline ARIMA/ARMA & 0.0002 & 1.0015 & 0.7990 & -1.9371 & 12.2046 \\
\hline ARIMA/Holt & -0.0001 & 1.1074 & 0.8836 & 0.4485 & 13.9419 \\
\hline ARMA/Holt & 0.0003 & 1.1071 & 0.8833 & -2.2674 & 13.9702 \\
\hline
\end{tabular}


Tabela 3: Medidas de Acurácia para séries temporais geradas a partir dos processos $\operatorname{ARFIMA}(p, d, q)$, quando $d=0.3, p=0, q=1, \theta_{1}=0.2$ e $n=1000$.

\begin{tabular}{lccccc}
\hline Modelos Ajustados & ME & RMSE & MAE & MPE & MAPE \\
\hline ARFIMA & 0.0006 & 0.9985 & 0.7967 & -1.0285 & 8.1506 \\
ARIMA & $\mathbf{0 . 0 0 0 0}$ & 1.0034 & 0.8002 & -1.0077 & 8.1807 \\
ARMA & 0.0001 & 0.9982 & 0.7969 & -1.0301 & 8.1384 \\
Holt & 0.0007 & 1.0993 & 0.8697 & -0.9323 & 8.8585 \\
\hline \multicolumn{5}{c}{ Combinação de Previsões - Variância Mínima } \\
\hline ARFIMA/ARIMA & -0.0001 & 0.9976 & 0.7964 & -1.0268 & 8.1329 \\
ARFIMA/ARMA & 0.0008 & 0.9968 & 0.7957 & -1.0193 & 8.1267 \\
ARFIMA/Holt & -0.0001 & 0.8740 & 0.6975 & -0.9154 & 7.1440 \\
ARIMA/ARMA & -0.0006 & 1.0023 & 0.7996 & -1.0200 & 8.1733 \\
ARIMA/Holt & -0.0003 & 0.8876 & 0.7084 & -0.8978 & 7.2432 \\
ARMA/Holt & -0.0001 & 0.8898 & 0.7101 & -0.9316 & 7.2750 \\
\hline \multicolumn{5}{c}{ Combinação de Previsões - Regressão } \\
\hline ARFIMA/ARIMA & 0.0005 & 0.9959 & 0.7953 & -1.0187 & 8.1292 \\
ARFIMA/ARMA & $\mathbf{0 . 0 0 0 0}$ & 0.9974 & 0.7960 & -1.0326 & 8.1400 \\
ARFIMA/Holt & 0.0003 & 0.8743 & 0.6977 & -0.9076 & 7.1291 \\
ARIMA/ARMA & 0.0003 & 0.9964 & 0.7954 & -1.0225 & 8.1295 \\
ARIMA/Holt & 0.0037 & 0.8856 & 0.7067 & -0.8535 & 7.2144 \\
ARMA/Holt & 0.0000 & 0.8890 & 0.7094 & -0.9246 & 7.2476 \\
\hline \multicolumn{5}{c}{ Combinação de Previsões - Média } \\
\hline ARFIMA/ARIMA & -0.0002 & 0.9968 & 0.7957 & -1.0200 & 8.1394 \\
ARFIMA/ARMA & 0.0002 & 0.9969 & 0.7957 & -1.0284 & 8.1302 \\
ARFIMA/Holt & 0.0001 & $\mathbf{0 . 8 4 6 3}$ & $\mathbf{0 . 6 7 3 6}$ & -0.8135 & $\mathbf{6 . 8 7 3 3}$ \\
ARIMA/ARMA & 0.0006 & 0.9968 & 0.7954 & -1.0134 & 8.1374 \\
ARIMA/Holt & 0.0004 & 0.8546 & 0.6806 & $\mathbf{- 0 . 8 0 4 9}$ & 6.9493 \\
ARMA/Holt & -0.0001 & 0.8524 & 0.6788 & -0.8199 & 6.9314 \\
\hline \multicolumn{5}{c}{ Fonte: Autores. }
\end{tabular}

Tabela 4: Medidas de Acurácia para séries temporais geradas a partir dos processos $\operatorname{ARFIMA}(p, d, q)$, quando $d=0.3, p=0, q=1, \theta_{1}=-0.2$ e $n=1000$.

\begin{tabular}{lccccc}
\hline Modelos Ajustados & ME & RMSE & MAE & MPE & MAPE \\
\hline ARFIMA & 0.0001 & 0.9979 & 0.7964 & -1.0469 & 8.1889 \\
ARIMA & -0.0005 & 1.0013 & 0.7989 & -1.0171 & 8.1995 \\
ARMA & 0.0001 & 0.9977 & 0.7964 & -1.0466 & 8.1839 \\
Holt & $\mathbf{0 . 0 0 0 0}$ & 1.0975 & 0.8739 & -0.8456 & 8.9308 \\
\hline \multicolumn{5}{c}{ Combinação de Previsões - Variância } & Mínima \\
\hline ARFIMA/ARIMA & -0.0005 & 0.9967 & 0.7953 & -1.0475 & 8.1866 \\
ARFIMA/ARMA & -0.0008 & 0.9977 & 0.7960 & -1.0515 & 8.1781 \\
ARFIMA/Holt & $\mathbf{0 . 0 0 0 0}$ & 0.9598 & 0.7659 & -1.0030 & 7.8554 \\
ARIMA/ARMA & -0.0002 & 0.9987 & 0.7967 & -1.0242 & 8.1886 \\
ARIMA/Holt & -0.0001 & 0.9488 & 0.7574 & -0.9539 & 7.7708 \\
ARMA/Holt & 0.0002 & 0.9613 & 0.7676 & -1.0059 & 7.8788 \\
\hline \multicolumn{5}{c}{ Combinação de Previsões - Regressão } \\
\hline ARFIMA/ARIMA & 0.0008 & 0.9980 & 0.7965 & -1.0286 & 8.1682 \\
ARFIMA/ARMA & $\mathbf{0 . 0 0 0 0}$ & 0.9966 & 0.7955 & -1.0480 & 8.1818 \\
ARFIMA/Holt & 0.0004 & 0.9581 & 0.7645 & -1.0036 & 7.8737 \\
ARIMA/ARMA & 0.0005 & 0.9960 & 0.7952 & -1.0333 & 8.1689 \\
ARIMA/Holt & 0.0046 & 0.9479 & 0.7568 & -0.9043 & 7.7581 \\
ARMA/Holt & $\mathbf{0 . 0 0 0 0}$ & 0.9618 & 0.7676 & -1.0141 & 7.8986 \\
\hline \multicolumn{5}{c}{ Combinação de Previsões - Média } \\
\hline ARFIMA/ARIMA & -0.0002 & 0.9979 & 0.7965 & -1.0308 & 8.1736 \\
ARFIMA/ARMA & -0.0003 & 0.9975 & 0.7963 & -1.0536 & 8.1892 \\
ARFIMA/Holt & 0.0007 & $\mathbf{0 . 8 3 4 8}$ & $\mathbf{0 . 6 6 5 5}$ & $\mathbf{- 0 . 7 5 5 7}$ & $\mathbf{6 . 8 1 9 7}$ \\
ARIMA/ARMA & 0.0002 & 0.9969 & 0.7949 & -1.0286 & 8.1638 \\
ARIMA/Holt & -0.0018 & 0.8349 & 0.6657 & -0.7659 & 6.8285 \\
ARMA/Holt & -0.0004 & 0.8357 & 0.6667 & -0.7739 & 6.8465 \\
\hline \multicolumn{5}{c}{ Fonte: Autores. } \\
\hline
\end{tabular}


Tabela 5: Medidas de Acurácia para séries temporais geradas a partir dos processos $\operatorname{ARFIMA}(p, d, q)$, quando $d=0.3, p=1, \phi_{1}=0.8 q=1, \theta_{1}=-0.2$ e $n=1000$.

\begin{tabular}{cccccc}
\hline Modelos Ajustados & ME & RMSE & MAE & MPE & MAPE \\
\hline ARFIMA & 0.0002 & 0.9979 & 0.7965 & -3.9851 & 19.9434 \\
ARIMA & $\mathbf{0 . 0 0 0 0}$ & 1.0106 & 0.8058 & 0.2030 & 17.2564 \\
ARMA & 0.0001 & 0.9969 & 0.7957 & -4.0811 & 20.5178 \\
Holt & 0.0009 & 1.0915 & 0.8706 & 0.5765 & 20.2942 \\
\hline \multicolumn{5}{c}{ Combinação de Previsões - Variância } & Mínima \\
\hline ARFIMA/ARIMA & 0.0002 & 0.9973 & 0.7958 & 5.8007 & 24.2898 \\
ARFIMA/ARMA & 0.0048 & 1.0030 & 0.8003 & -30.4502 & 45.4219 \\
ARFIMA/Holt & 0.0002 & 1.4316 & 1.1431 & -5.9641 & 29.9762 \\
ARIMA/ARMA & 0.0002 & 1.0110 & 0.8065 & $\mathbf{0 . 0 8 6 3}$ & $\mathbf{1 7 . 0 5 2 5}$ \\
ARIMA/Holt & $\mathbf{0 . 0 0 0 0}$ & 1.4355 & 1.1455 & -0.1687 & 26.1373 \\
ARMA/Holt & -0.0002 & 1.4300 & 1.1412 & -6.4398 & 32.0171 \\
\hline \multicolumn{5}{c}{ Combinação de Previsões - Regressão } \\
\hline ARFIMA/ARIMA & 0.0045 & 0.9970 & 0.7956 & -4.2765 & 20.8610 \\
ARFIMA/ARMA & 0.0001 & 0.9967 & 0.7957 & -0.8420 & 19.9359 \\
ARFIMA/Holt & 0.0006 & 1.4319 & 1.1433 & -1.3433 & 24.5153 \\
ARIMA/ARMA & 0.0031 & 0.9965 & $\mathbf{0 . 7 9 4 9}$ & -2.6315 & 17.9124 \\
ARIMA/Holt & 0.0477 & 1.4343 & 1.1449 & 2.1888 & 27.7956 \\
ARMA/Holt & -0.0008 & 1.4279 & 1.1398 & 7.0087 & 33.1381 \\
\hline \multicolumn{5}{c}{ Combinação de Previsões - Média } \\
\hline ARFIMA/ARIMA & -0.0003 & 1.0009 & 0.7981 & -0.5809 & 21.3508 \\
ARFIMA/ARMA & 0.0002 & $\mathbf{0 . 9 9 6 4}$ & 0.7954 & -2.0612 & 17.6468 \\
ARFIMA/Holt & 0.0004 & 1.2619 & 1.0068 & -0.3275 & 23.5462 \\
ARIMA/ARMA & $\mathbf{0 . 0 0 0 0}$ & 0.9997 & 0.7978 & -3.8775 & 20.1004 \\
ARIMA/Holt & 0.0009 & 1.2627 & 1.0073 & -25.1495 & 49.3979 \\
ARMA/Holt & $\mathbf{0 . 0 0 0 0}$ & 1.2593 & 1.0050 & -0.7913 & 22.2157 \\
\hline \multicolumn{5}{c}{ Fonte: Autores. }
\end{tabular}

\section{Aplicação a Dados Reais}

A seguir analisamos a série temporal do valor do ativo do Banco Bradesco SA na hora do fechamento da bolsa de valores utilizando a metodologia desenvolvida neste trabalho. A etapa da obtenção de dados neste artigo, consistiu em resgatar dados históricos do site Yahoo Finanças (https://br.financas.yahoo.com/). Serão utilizadas as 1853 observações diárias disponíveis, de $04 / 01 / 2010$ a $27 / 06 / 2017$. Os dados foram acessados em $04 / 08 / 2017$. O uso deste período se deve ao fato de o período de dados mais completo disponíveis na internet e que foram encontrados pelos autores.

Uma vez que se busca uma técnica adequada prever o valor do ativo do Banco Bradesco SA na hora do fechamento da bolsa de valores, obteve-se as previsões utilizando os modelos de Suavização Exponencial, $\operatorname{ARFIMA}(p, d, q), \operatorname{ARIMA}(p, d, q)$ e $\operatorname{ARMA}(p, q)$ e suas respectivas combinações, utilizando dois modelos base.

A Figura 1 apresenta o gráfico da séries temporal e da função de autocorrelação amostral. Podemos perceber, pelo gráfico da série temporal e pela sua função de autocorrelação amostral que a série pode ser tratada como estacionária com a propriedade de longa dependência, mas também pode ser tratada como não estacionária. Foram aplicados os testes de raiz unitária de Dickey-Fuller, que apresentou $p$-valor $=0.1551$, e de Phillips-Perron, que apresentou $p$-valor $=$ 
0.04145. Ambos testam as hipóteses: $H_{0}$ : série temporal não estacionária versus $H_{1}$ : série temporal estacionária. Os testes foram realizados utilizando, respectivamente, as rotinas adf.test e pp.test, do pacote tseries do R. Os resultados dos dois testes de raiz unitária foram inconclusivos quanto a estacionariedade da série temporal.

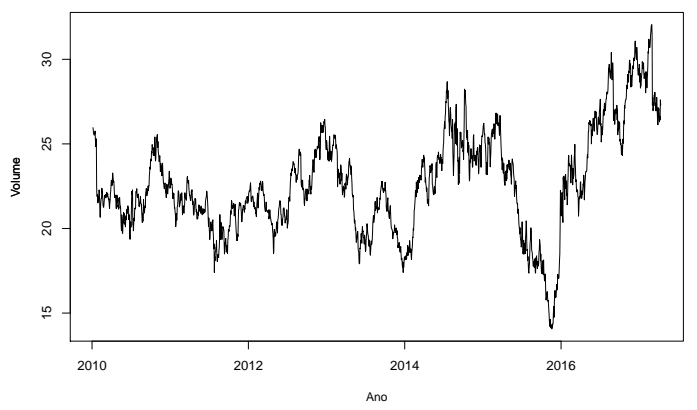

(a) Valor de Fechamento dos Ativos do Banco Bradesco SA.

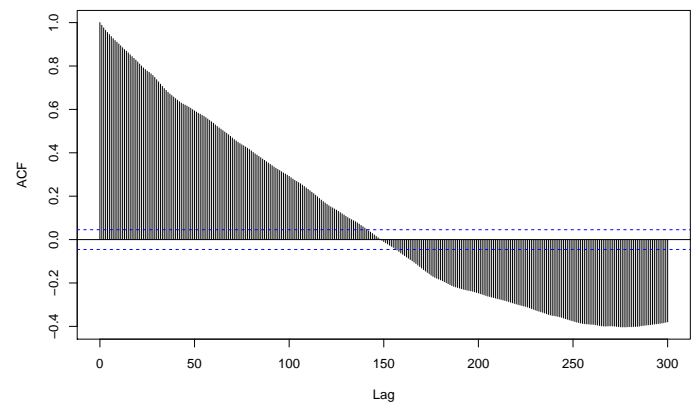

(b) $\mathrm{ACF}$

Figura 1: (a) Valor de Fechamento dos Ativos do Banco Bradesco SA, de 04/01/2010 a 27/06/2017. (b) ACF Amostral.

Fonte: Autores.

A seguir apresentamos os modelos ajustados a série temporal do valor do ativo do Banco Bradesco SA na hora do fechamento da bolsa de valores.

Modelo 1 - $\operatorname{ARFIMA}(p, d, q)$, com $\widehat{d}=0.0458, p=1$, onde $\widehat{\phi}_{1}=0.9890$ e $q=0$. Para este modelo, obtivemos uma variância estimada dos resíduos igual a $\widehat{\sigma}^{2}=0.2104$, Critério de Informação de Akaike $A I C=2375.877$ e $p-$ valor $=0.6571$ para o teste de Box - Pierce para os resíduos.

Modelo 2 - $\operatorname{ARIMA}(p, d, q)$, com $p=0=q$ e $d=1$. Para este modelo, obtivemos uma variância estimada dos resíduos igual a $\widehat{\sigma}^{2}=0.2115$, Critério de Informação de Akaike $A I C=2381.62$ e $p-$ valor $=0.4938$ para o teste de Box - Pierce para os resíduos.

Modelo 3 - $\operatorname{ARMA}(p, q)$, com $p=1, q=0$, onde $\widehat{\phi}_{1}=0.9790$. Para este modelo, obtivemos uma variância estimada dos resíduos igual a $\widehat{\sigma}^{2}=0.2125$, Critério de Informação de Akaike $A I C=2348.86$ e $p-$ valor $=0.6587$ para o teste de Box - Pierce para os resíduos.

Modelo 4 - Modelo de Suavização Exponencial (Modelo Linear de Holt): as estimativas para os parâmetros do modelo são: $\widehat{\alpha}=0.9856, \widehat{\beta}=0.00564$. Para este modelo, obtivemos 
uma variância estimada dos resíduos igual a $\widehat{\sigma}^{2}=0.212891$ e $p-$ valor $=0.9738$ para o teste de Box - Pierce para os resíduos.

As Tabelas 6 a 9 a seguir apresentam as medidas de acurácia dos para as previsões utilizando os Modelos 1 a 4 ajustados e a combinação de previsões combinadas dois a dois. Analisando tais tabelas, verificamos que o Modelo 2 apresenta menor ME, em valor absoluto. A combinação das previsões dos Modelos 2 e 3, por variância mínima, apresenta menor MAE e MAPE, com $\widehat{w}=0.9110477$. A combinação das previsões dos Modelos 1 e 3, por regressão, apresenta menor RMSE, com $\widehat{\beta}_{1}=-15.86, \widehat{\beta}_{2}=16.86$ e $R^{2}$ ajustado de 0.986 . Por último a combinação de previsões dos modelos 3 e 4, por média, apresenta menor MPE, em valor absoluto.

Tabela 6: Medidas de Acurácia dos Modelos 1 a 4.

\begin{tabular}{cccccc}
\hline Modelo Ajustado & ME & RMSE & MAE & MPE & MAPE \\
\hline Modelo 1 & 0.000953 & 0.458560 & 0.334767 & -0.036906 & 1.485480 \\
Modelo 2 & $\mathbf{0 . 0 0 0 0 1 4}$ & 0.459645 & 0.334200 & -0.020778 & 1.482779 \\
Modelo 3 & -0.001027 & 0.458546 & 0.334766 & -0.045816 & 1.485664 \\
Modelo 4 & 0.013157 & 0.461464 & 0.336099 & 0.047475 & 1.491103 \\
\hline \multicolumn{5}{c}{ Fonte: Autores. }
\end{tabular}

Tabela 7: Medidas de Acurácia para as Combinações de Previsão: Variância Mínima.

\begin{tabular}{cccccc}
\hline Combinação de Previsão & ME & RMSE & MAE & MPE & MAPE \\
\hline Modelos 1 e 2 & 0.000932 & 0.458547 & 0.334626 & -0.035013 & 1.484809 \\
Modelos 1 e 3 & 0.040178 & 0.460744 & 0.336245 & 0.139534 & 1.488609 \\
Modelos 1 e 4 & 0.002594 & 0.458586 & 0.334513 & -0.025046 & 1.484326 \\
Modelos 2 e 3 & 0.000575 & 0.459439 & $\mathbf{0 . 3 3 4 1 7 0}$ & -0.020068 & $\mathbf{1 . 4 8 2 6 1 2}$ \\
Modelos 2 e 4 & 0.000432 & 0.459882 & 0.334482 & -0.019219 & 1.484048 \\
Modelos 3 e 4 & 0.000922 & 0.458579 & 0.334516 & -0.032573 & 1.484486 \\
\hline \multicolumn{5}{c}{ Fonte: Autores. }
\end{tabular}

Tabela 8: Medidas de Acurácia para as Combinações de Previsão: Média.

\begin{tabular}{cccccc}
\hline Combinação de Previsão & ME & RMSE & MAE & MPE & MAPE \\
\hline Modelos 1 e 2 & 0.000843 & 0.458765 & 0.334233 & -0.027228 & 1.482913 \\
Modelos 1 e 3 & -0.000037 & 0.458551 & 0.334765 & -0.041361 & 1.485565 \\
Modelos 1 e 4 & 0.006950 & 0.459091 & 0.334497 & 0.004856 & 1.483973 \\
Modelos 2 e 3 & -0.000148 & 0.458760 & 0.334228 & -0.031683 & 1.482968 \\
Modelos 2 e 4 & 0.006973 & 0.460303 & 0.334908 & 0.015062 & 1.485829 \\
Modelos 3 e 4 & 0.005966 & 0.459076 & 0.334481 & $\mathbf{0 . 0 0 0 4 2 6}$ & 1.483962 \\
\hline
\end{tabular}

A Figura 2 apresenta as Predições e as Previsões da Série Temporal do valor do ativo do Banco Bradesco SA na hora do fechamento da bolsa de valores, utilizando o modelo e as combinações de previsão com menores medidas de acurácia. Observa-se que os modelos e combinação de previsões captam o comportamento dos dados e as previsões apresentadas possuem pouca variação entre si. 
Tabela 9: Medidas de Acurácia para as Combinações de Previsão: Regressão.

\begin{tabular}{cccccc}
\hline Combinação de Previsão & ME & RMSE & MAE & MPE & MAPE \\
\hline Modelos 1 e 2 & 0.000411 & 0.458546 & 0.334630 & -0.037350 & 1.484872 \\
Modelos 1 e 3 & -0.000624 & $\mathbf{0 . 4 5 8 4 3 5}$ & 0.334743 & -0.046644 & 1.485753 \\
Modelos 1 e 4 & 0.000971 & 0.458583 & 0.334521 & -0.032412 & 1.484500 \\
Modelos 2 e 3 & 0.000365 & 0.458533 & 0.334629 & -0.037762 & 1.484887 \\
Modelos 2 e 4 & 0.004310 & 0.459863 & 0.334531 & -0.002468 & 1.484046 \\
Modelos 3 e 4 & 0.000958 & 0.458579 & 0.334523 & -0.032533 & 1.484513 \\
\hline \multicolumn{5}{c}{ Fonte: Autores. }
\end{tabular}

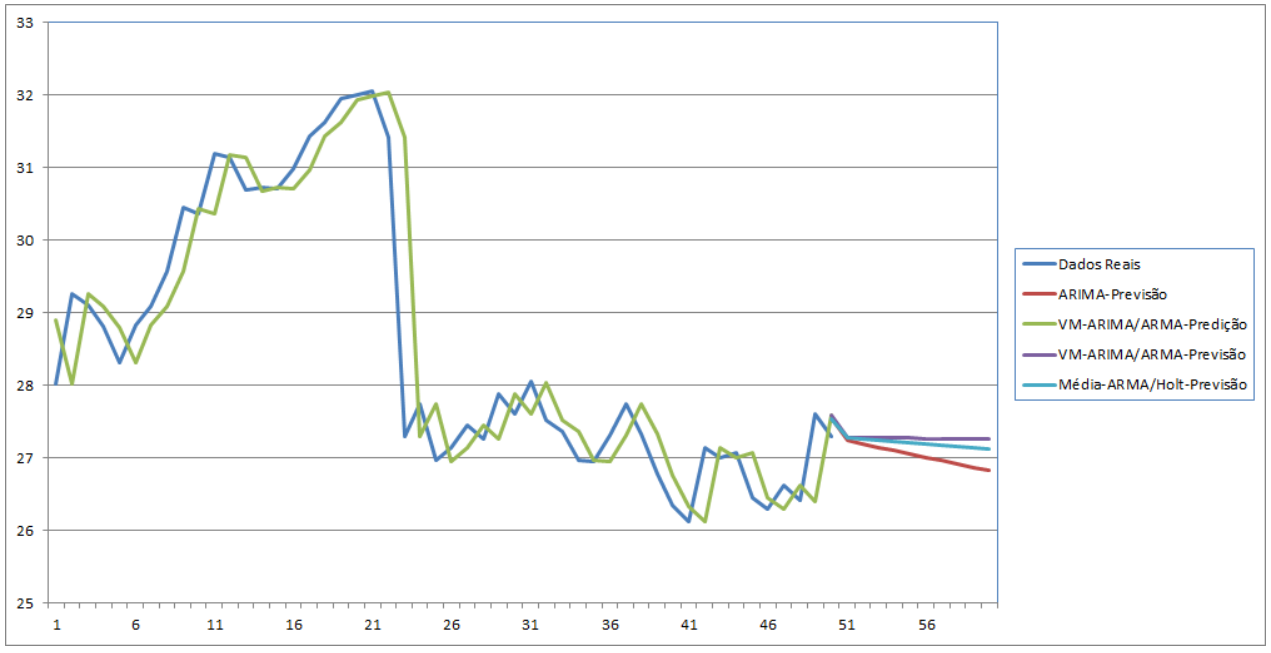

Figura 2: Previsão da Série Temporal do valor do ativo do Banco Bradesco SA na hora do fechamento da bolsa de valores.

Fonte: Autores.

\section{Considerações Finais}

Ao concluirmos nossas análises, podemos verificar que na maioria dos casos as menores medidas de acurácia, nas simulações de Monte Carlo, foram obtidas através de combinação de previsões. Cabe ressaltar que, em alguns casos as menores medidas médias foram obtidas quando a previsão foi feita somente com um modelo, e nestes casos, em apenas uma situação o modelo $\operatorname{ARFIMA}(p, d, q)$ obteve menores medidas de acurácia médias. Ou seja, mesmo tendo uma série gerada a partir deste processo, as combinações exerceram melhor papel preditivo do que o modelo propriamente dito. O mesmo pode ser observado na aplicação em dados reais, uma vez que quatro das cinco menores medidas de acurácia são obtidas a partir de combinação de previsões.

Desta forma, é possível concluir com base nas simulações de Monte Carlo e aplicação realizada neste artigo, que mesmo tendo um problema na especificação do modelo, como é o caso da aplicação utilizando a série temporal do valor do ativo do Banco Bradesco SA na hora do fechamento da bolsa de valores, podemos obter boas previsões.

Neste caso, a combinação de previsões pode ser uma ótima alternativa para aperfeiçoar a 
previsão, uma vez que, como na maioria dos casos apresentados na seção de simulações de Monte Carlo deste trabalho, é possível aprimorar a capacidade preditiva do modelo visto que as mesmas apresentam menores medidas de acurácia quando comparadas com previsões geradas utilizandose apenas um modelo.

\section{Referências}

Abraham, B. e Ledolter, J. (2009). Statistical methods for forecasting, volume 234. John Wiley \& Sons.

Bates, J. M. e Granger, C. W. (1969). The combination of forecasts. Or, pages 451-468.

Bisognin, C. (2007). Estimação e previsão em processos $\operatorname{SARFIMA}(p, d, q) \times(P, D, Q)_{s}$ na presença de outlier. Tese de Doutorado. Universidade Federal do Rio grande do Sul. Programa de Pós-Graduação em Matemática. Porto Alegre. PhD thesis.

Box, G. E. e Jenkins, G. M. (1976). Time series analysis, control, and forecasting. San Francisco, CA: Holden Day, 3226(3228):10.

Costantini, M. e Pappalardo, C. (2010). A hierarchical procedure for the combination of forecasts. International journal of forecasting, 26(4):725-743.

De Menezes, L. M., Bunn, D. W., e Taylor, J. W. (2000). Review of guidelines for the use of combined forecasts. European Journal of Operational Research, 120(1):190-204.

Gardner, G., Harvey, A. C., e Phillips, G. D. (1980). Algorithm as 154: An algorithm for exact maximum likelihood estimation of autoregressive-moving average models by means of kalman filtering. Journal of the Royal Statistical Society. Series C (Applied Statistics), 29(3):311-322.

Geweke, J. e Porter-Hudak, S. (1983). The estimation and application of long memory time series models. Journal of time series analysis, 4(4):221-238.

Granger, C. W. e Joyeux, R. (1980). An introduction to long-memory time series models and fractional differencing. Journal of time series analysis, 1(1):15-29.

Granger, C. W. e Ramanathan, R. (1984). Improved methods of combining forecasts. Journal of forecasting, 3(2):197-204.

Haslett, J. e Raftery, A. E. (1989). Space-time modelling with long-memory dependence: Assessing ireland's wind power resource. Applied Statistics, pages 1-50. 
Hosking, J. R. (1981). Fractional differencing. Biometrika, 68(1):165-176.

Hosking, J. R. (1984). Modeling persistence in hydrological time series using fractional differencing. Water resources research, 20(12):1898-1908.

Khandakar, Y. e Hyndman, R. J. (2008). Automatic time series forecasting: the forecast package for r. Journal of Statistical Software, 27(03).

Lima, R. C., Góis, M. R., e Ulises, C. (2007). Previsão de preços futuros de commodities agrícolas com diferenciações inteira e fracionária, e erros heteroscedásticos. Brazilian Journal of Rural Economy and Sociology (RESR), 45(3).

Makridakis, S., Wheelwright, S. C., e Hyndman, R. J. (1998). Forecasting methods and applications. John wiley \& sons.

Morettin, P. A. e Toloi, C. (2006). Análise de séries temporais. Blucher.

Pellegrini, F. R. (2000). Metodologia para implementação de sistemas de previsão de demanda. Dissertaçãoo de Mestrado. Porto Alegre: UFRGS.

Queiroz, F. F. (2016). Estudo sobre má especificação na família de posição e escala. Monografia (Especialização) - Curso de Estatística. Natal: UFRN.

Werner, L. (2005). Um modelo composto para realizar previsão de demanda através da integração da combinação de previsões e do ajuste baseado na opinião. Tese de Doutorado. Universidade Federal do Rio grande do Sul. Programa de Pós-Graduação em Engenharia de Produção. Porto Alegre. $\mathrm{PhD}$ thesis. 